\title{
Production of (R)-3-hydroxybutyric acid by Burkholderia cepacia from wood extract hydrolysates
}

Yuanzhen Wang and Shijie Liu*

\begin{abstract}
(R)-hydroxyalkanoic acids (R-HAs) are valuable building blocks for the synthesis of fine chemicals and biopolymers because of the chiral center and the two active functional groups. Hydroxyalkanoic acids fermentation can revolutionize the polyhydroxyalkanoic acids (PHA) production by increasing efficiency and enhancing product utility. Modifying the fermentation conditions that promotes the in vivo depolymerization and secretion to fermentation broth in wild type bacteria is a novel and promising approach to produce R-HAs. Wood extract hydrolysate (WEH) was found to be a suitable substrate for R-3-hydroxybutyric acid (R-3-HB) production by Burkholderia cepacia. Using Paulownia elongate WEH as a feedstock, the R-3-HB concentration in fermentation broth reached as high as $14.2 \mathrm{~g} / \mathrm{L}$ after 3 days of batch fermentation and the highest concentration of $16.8 \mathrm{~g} / \mathrm{L}$ was obtained at day 9 . Further investigation indicated that the composition of culture medium contributed to the enhanced R-3-HB production.
\end{abstract}

Keywords: (R)-3-hydroxybutyric acid; Burkholderia cepacia; Wood extract hydrolysate

\section{Introduction}

(R)-hydroxyalkanoic acids (R-HAs) are valuable chiral building blocks for synthesis of fine chemicals and pharmaceuticals, such as antibiotics, amino acids, vitamins, food supplements, cosmetics and many others (Chen and Wu 2005; Lee et al. 1999; Ren et al. 2010). In addition, R-HAs can be used as precursors to produce homopolymers and "tailor-made" copolymers as well as to overcome the challenge of producing high molecular weight polymers through controlled in vitro polymerization (Ren et al. 2010). The R-HAs contain at least one chiral center and two active functional groups: a hydroxyl group and a carboxyl group (Lee and Park 2009). HAs being enantiomerically pure is important for their applications. For example, a chiral pharmaceutical possesses advantages including improved safety, enhanced efficiency, lower dosage and reduced side effects compared to traditionally synthesized compounds (Chen and $\mathrm{Wu}$ 2005; Ren et al. 2010).

Polyhydroxyalkanoates (PHAs) are a family of polyesters commonly known to be synthesized by bacteria as

\footnotetext{
* Correspondence: sliu@esf.edu

Department of Paper and Bioprocess Engineering, SUNY College of

Environmental Science and Forestry, 1 Forestry Drive, Syracuse, NY 13210, USA
}

intracellular carbon and energy storage compounds (Wang and Liu 2013). PHAs from microbial sources are all composed of chiral (R)-hydroxyalkanoic acids (Chen and Wu 2005; Lee and Park 2009; Lee et al. 1999). To date, approximately 150 R-HA monomers have been identified (Steinbüchel and Lütke-Eversloh 2003). Industrial production of PHA has been based on the intracellular synthesis, which is different from polylactic acid (PLA) production (Buyondo and Liu 2011). Lactic acid is produced as an extracellular product, and then lactic acid is chemically polymerized to PLA. The polymer properties can be adjusted to some degree by adjusting the degree of polymerization. Apart from limited utility and/or narrow distribution of properties, intracellular PHA incurs significant cost in extraction, separation and purification (Chen 2009; Choi and Lee 1999). It is therefore desirable to examine the potential for the extracellular production/modification of PHAs to expand their utility.

Currently, methods for production of R-HAs include chemical synthesis, biocatalysis, chemical or enzymatic degradation of biologically synthesized Polyhydroxyalkanoates (PHAs), in vivo depolymerization of PHAs, and biotechnological production by metabolic pathway engineering 
(Chen and Wu 2005; Ren et al. 2010). Direct chemical synthesis of R-HAs has many limitations such as the requirement of pure substrate and expensive catalysts (Ren et al. 2005; Ren et al. 2010). Using microorganisms as biocatalysts for R-HA production often gives low yields and enantiomeric excesses (Ren et al. 2005). In vivo depolymerization of PHAs is disadvantageous due to the low efficiency (de Roo et al. 2002; Kawata et al. 2012). In vivo depolymerization of PHA requires the biomass with PHA accumulated to be harvested from fermentation broth first, and then PHA degradation is enhanced by modifying the $\mathrm{pH}$ or temperature (Lee et al. 1999; Ren et al. 2005). Metabolic pathway engineering has been employed to produce R-HAs by overexpression of depolymerase and/or under-expression of synthase or dehydrogenase, but it is still at a stage in need of practical use (Chung et al. 2009; Park et al. 2004; Romano et al. 2005; Sandoval et al. 2005).

Production of R-HAs by wild-type bacteria fermentation through modifying the culture conditions is a very promising and innovative approach (Kawata et al. 2012; Ren et al. 2010). R-HA is then an extracellular product, which is more efficient because it can be harvested directly from fermentation broth. To date, there is very little research exploring the direct fermentation method for R-HA production. Applying successive aerobic and microaerobic culture condition has been reported to increase the extracellular accumulation of R-HAs by Halomonas sp. KM-1 in $200 \mathrm{~mL}$ Erlenmeyer flasks culture (Kawata et al. 2012).

In our previous publications, the production of polyhydroxybutyric acids (PHB) by Burkholderia cepacia was reported using wood extract hydrolysate (Keenan et al. 2004; Liu 2012; Wang et al. 2012). In this paper, we investigated a novel approach for the production of (R)-3hydroxybutyric acid (R-3-HB) by Burkholderia cepacia fermentation through alteration of culture conditions and using wood extract hydrolysate as the fermentation substrate. To the best of our knowledge, it is the first time reporting utilizing wild type Burkholderia cepacia to directly produce R-3-HB.

\section{Materials and methods}

\section{Preparation of Acer saccharum WEH}

Hot water extraction on Acer saccharum woodchips was carried out at $160^{\circ} \mathrm{C}$ for $2 \mathrm{~h}$. The resulting suspension was then subjected to a membrane filtration to remove the suspended solids and to concentrate the wood extract. Dilute acid hydrolysis of the wood extract was then performed with sulfuric acid $(1.5 \mathrm{wt} \%)$ at $120^{\circ} \mathrm{C}$ for $30 \mathrm{~min}$. Wood extract hydrolysate was centrifuged to remove solid deposits before neutralized by calcium hydroxide and again centrifuged to remove the precipitate (CEPA centrifuge Z81G). Ten times dilution of the hydrolysate before nano-filtration was performed and repeated twice in order to remove and to recover fermentation inhibiting compounds such as acetic acid, furfural, and 5hydroxymethylfurfural (Shupe and Liu 2012b; Sun and Liu 2012).

\section{Preparation of Paulownia elongata WEH}

Four year old Paulownia elongata wood logs were obtained from Fort Valley State University, Fort Valley in Georgia and were chipped with bark-on and screened to a uniform size of $2.5 \times 2.0 \times 0.5 \mathrm{~cm}^{3}$. A $1.84 \mathrm{~m}^{3} \mathrm{di}-$ gester with external steam heating system was used to carry out the hot-water extraction. Water to solid ratio was 7.69:1. The extraction was conducted nominally at $160^{\circ} \mathrm{C}$ for $2 \mathrm{~h}$ (Yan et al. 2013). The extraction liquor was devoid of solids via ultra-filtration and then concentrated via nano-filtration. Dilute acid hydrolysis of the concentrated wood extract was then performed with mixed acids: hydrochloric acid (0.1 wt\%), nitric acid $(0.1 \mathrm{wt} \%)$, and sulfuric acid $(0.8 \mathrm{wt} \%)$ at $121^{\circ} \mathrm{C}$ for $60 \mathrm{~min}$. The hydrolysate was devoid of solids through paper filtration before neutralization with calcium hydroxide and sodium hydroxide. The neutralized wood extract hydrolysate was then filtered to remove solids with filter paper. Ten times dilution of the hydrolysate followed by nano-filtration was performed and repeated in order to remove and to recover fermentation inhibiting compounds such as acetic acid, furfural, and 5-hydroxymethylfurfural (Shupe and Liu 2012b; Sun and Liu 2012).

\section{Batch fermentation}

Bukerholderia cepacia ATCC 17795 was purchased from American Type Culture Collection. The batch fermentations of $B$. cepacia utilizing untreated $50 \mathrm{~g} / \mathrm{L}$ xylose, $50 \mathrm{~g} / \mathrm{L}$ glucose, $50 \%$ sugar maple WEH, and $50 \%$ Paulownia elongata WEH were performed in a $1.0 \mathrm{~L}$ bioreactor (Bioflo 110, Newbrunswick, USA). For the experiments of simulating Paulownia elongata WEH by adding extra nitrate and chloride ions to xylose solution, calcium nitrate and calcium chloride was added at the same amount as introduced by dilute acid hydrolysis.

The working volume was $500 \mathrm{~mL}$, contained $50 \mathrm{mg}$ $\mathrm{CaCl}_{2}, 30 \mathrm{mg} \mathrm{NH} \mathrm{NH}_{4} \mathrm{Fe}(\mathrm{III})$ citrate, $100 \mathrm{mg} \mathrm{MgSO}_{4}$, and 0.07\% levulinic acid (Bertrand et al. 1990; Keenan et al. 2004). $\mathrm{Na}_{2} \mathrm{HPO}_{4}$ and $\mathrm{Na}_{2} \mathrm{PO}_{4}$ were added with a weight ratio of 1:1.35 to achieve a final $0.02 \mathrm{~mol} / \mathrm{L}$ phosphate ion concentration. The nitrogen source is limited by reducing the concentration of $\left(\mathrm{NH}_{4}\right)_{2} \mathrm{SO}_{4}$ to $1.5 \mathrm{~g} / \mathrm{L}$ (Keenan et al. 2004). The $\mathrm{pH}$ was maintained at 7.0 by adding $5 \mathrm{M}$ $\mathrm{NaOH}$. Excessive foaming was detected by a foam probe and defoaming was carried out by addition of silicone based antifoam. 
The biomass is harvested from fermentation broth by centrifugation at $4000 \times \mathrm{g}$ for $10 \mathrm{~min}$ followed by freeze drying and then weighted to obtain the dried weight of the biomass. The freeze dried biomass was suspended into $50 \mathrm{~mL}$ chloroform (5\% wt/vol) and incubated in $250 \mathrm{~mL}$ sealed glass flasks at $60^{\circ} \mathrm{C}$ for $24 \mathrm{~h}$. Chloroform solution was obtained by filtration with whatman \#1 filter paper, and then methanol was added to the PHB solution at ration of $5: 1(\mathrm{vol} / \mathrm{vol})$. PHB was therefore precipitated and harvested by centrifugation at $4000 \times \mathrm{g}$ for $20 \mathrm{~min}$. Polymer films were prepared by re-dissolving polymer with chloroform in $10 \mathrm{~cm}$ glass Petri dishes followed by evaporating chloroform in a fume hood for overnight. The PHB polymer was then weighted to obtain the PHB yield.

\section{Analytical methods}

Sugar concentrations in WEH were analyzed using nuclear magnetic resonance (NMR) spectroscopy (AVANCE, Bruker BioSpin co.) operated at $600 \mathrm{MHz}$. A modified two dimensional (2D) heteronuclear single quantum coherence (HSQC) method was used to determine the sugar concentration (Shupe et al. 2012; Shupe and Liu 2012a).

Characterization and quantification of (R)-3-hydrobutyric acid was obtained by analyzing ${ }^{1} \mathrm{H}$ nuclear magnetic resonance spectroscopy (AVANCE 600, Bruker BioSpin co.) operated at $600 \mathrm{MHz}$. The concentration of $\mathrm{R}-3-\mathrm{HB}$ was calculated by integrating the peak area at $1.12 \mathrm{ppm}$ corresponding to the peak area of internal standard trimethylamine (TMA) with known concentration of $2.28 \mathrm{~g} / \mathrm{L}$.

$$
\frac{P_{H B}}{P_{T M A}}=\frac{M_{H B} \times 3}{M_{T M A} \times 9}
$$

Where $\mathrm{P}_{\mathrm{HB}}$ and $\mathrm{P}_{\mathrm{TMA}}$ are the integrated peak area of $\mathrm{R}-3-\mathrm{HB}$ at $1.12 \mathrm{ppm}$ and TMA at $2.90 \mathrm{ppm}$, and $\mathrm{M}_{\mathrm{HB}}$ and $\mathrm{M}_{\mathrm{TMA}}$ are mole concentrations of $\mathrm{R}-3-\mathrm{HB}$ and TMA respectively.

\section{Results}

Batch fermentation using wood extract hydrolysates (WEH) NMR 2D HSQC spectrums with chemical shifts ranging from 4.1 to $6.0 \mathrm{ppm}$ for ${ }^{1} \mathrm{H}$ (f2) and 91 to $106 \mathrm{ppm}$ for ${ }^{13} \mathrm{C}$ (f1) was obtained for sugar concentration determination in wood extract hydrolysates (WEH) as shown in Figure 1. The concentrations of monosaccharides were calculated using the method reported previously (Shupe et al. 2012). First the peaks of each monosaccharaide and oligomers were identified in the 2D-HSQC spectrum and then their concentrations were calculated using the normalized peak areas using glucosamine $(5.49,92.10)$ as the internal standard. Approximate peak locations of the main components in WEH are: $\alpha$-xylose $(5.20,95.06), \alpha-$ glucose $(5.23,94.91)$, $\alpha$-galactose $(5.27,95.12)$.

In Paulownia elongate WEH, there are $12.3 \mathrm{~g} / \mathrm{L}$ galactose, $19.5 \mathrm{~g} / \mathrm{L}$ glucose, and $39.5 \mathrm{~g} / \mathrm{L}$ xylose before $B$. серacia batch fermentation (Figure 1A). Xylose is the most abundant monosaccharide in Acer saccharum WEH, which has a concentration of $47.2 \mathrm{~g} / \mathrm{L}$ before batch fermentation (Figure 1C). After 10 and 11 days of B. cepacia fermentation, almost all of the monosaccharides are consumed (Figure $1 \mathrm{~B}$ and D). Xylo-oligomers $(4.47,104.3)$ were also detected in Paulownia elongate WEH as shown in Figure 1A. However, these oligomers were consumed to a large extent after 11 days of fermentation (Figure $1 C$ ), which indicated the ability of $B$. cepacia to utilize oligosaccharides.

\section{Characterization and quantification of R-3-HB}

The proton NMR spectrum of a regent 3-HB in water solution is shown in Figure 2. The resonance peaks at $1.2 \mathrm{ppm}, 2.5 \mathrm{ppm}$, and $4.2 \mathrm{ppm}$ correspond to the labeled protons on the 3-HB molecule. These resonance peaks of 3-HB were also identified on the proton spectrum of $B$. cepacia fermentation broth (Figure 3 ). Because the peaks at $2.5 \mathrm{ppm}$ and $4.2 \mathrm{ppm}$ overlap with resonance peaks of other compounds in the fermentation broth, we only integrate peak area of the double peak located at $1.2 \mathrm{ppm}$ to quantify the concentration of $3-\mathrm{HB}$.

A linear regression line $\left(y=0.1134 x, R^{2}=0.9937\right)$ was obtained using the regent $3-\mathrm{HB}$ standard solutions (Figure 4). The normalized peak area of $3-\mathrm{HB}$ at $1.2 \mathrm{ppm}$ based on internal standard (trimethylamine, TMA) showed a positive linear correlation with the concentration of 3-HB at the range of $0 \mathrm{~g} / \mathrm{L}$ to $20 \mathrm{~g} / \mathrm{L}$. To ascertain the quantitative nature of the proton NMR, Figure 5 shows a comparison of the concentration as determined from NMR reading and those of standard solutions. One can observe from Figure 5 that the concentration of R-3-HB determined by NMR agrees very well with the actual concentration in the solution.

\section{R-3-HB production from WEH}

From the proton spectrum of $B$. cepacia fermentation broth using Paulownia elongate WEH as a substrate (Figure 3), right shifting of resonance peaks of 3-HB is observed. For example, the $\mathrm{CH}$ resonance peak located at $1.20 \mathrm{ppm}$ for the regent $3-\mathrm{HB}$, but it shifted slightly right to $1.12 \mathrm{ppm}$ for the $3-\mathrm{HB}$ produced in the fermentation broth. This resonance peak shifting is most probably resulted from neutralization of acids by sodium hydroxide. Sodium hydroxide was dosed into the medium automatically and consistently in order to maintain the $\mathrm{pH}$ at 7.0 in the fermentation.

In addition to $3-\mathrm{HB}$, acetic acid which has a resonance peak at $1.90 \mathrm{ppm}$, was also identified in the fermentation 


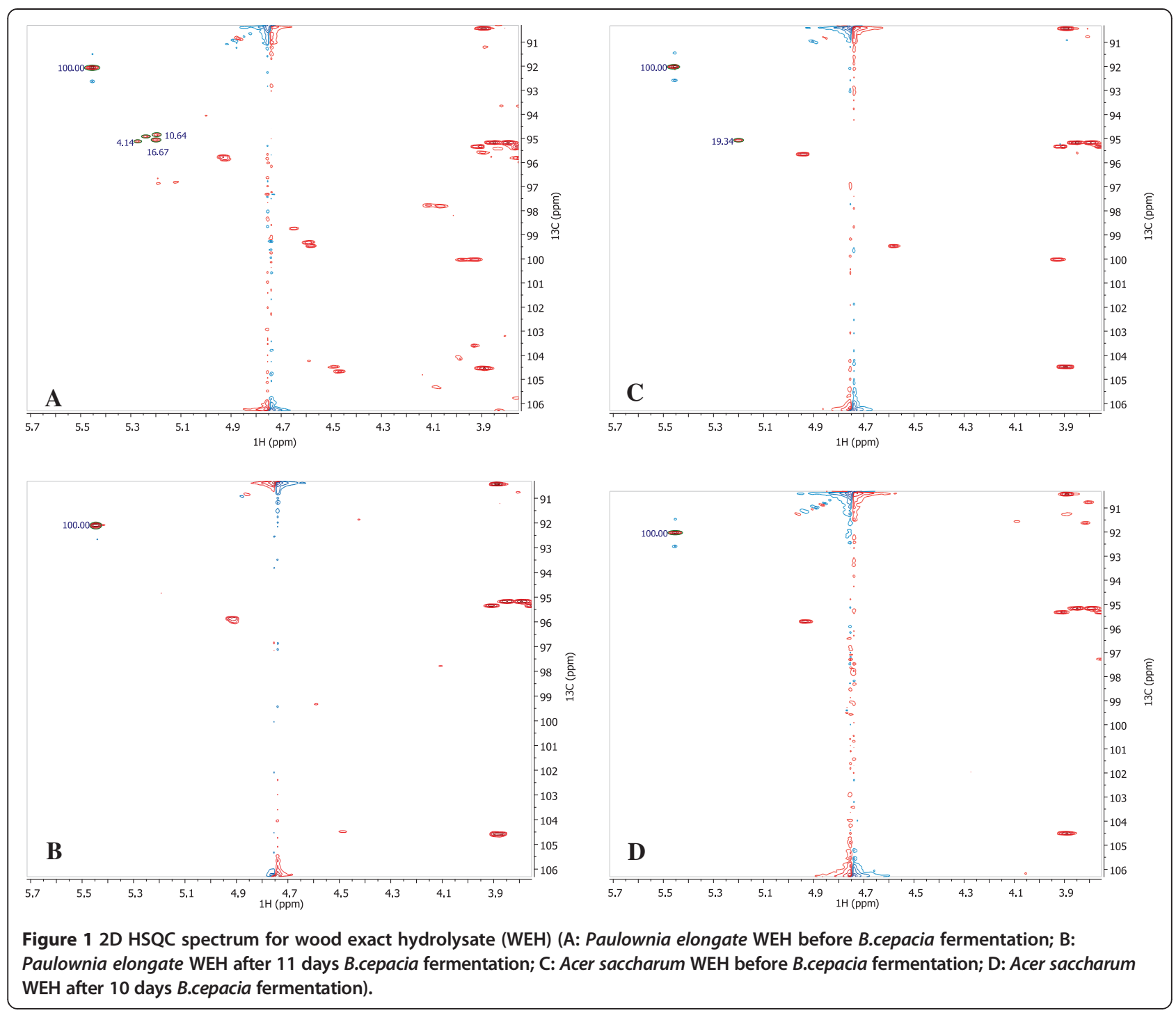

broth from the proton spectrum (Figure 3). The accumulation of acetic acid started at day 3 and reached the peak concentration of $16.6 \mathrm{~g} / \mathrm{L}$ at day $11(240 \mathrm{~h})$, and then decreased to $12.4 \mathrm{~g} / \mathrm{L}$ at day $12(262 \mathrm{~h})$. The metabolism of acetic acid production by B. cepacia is beyond the research scope of this paper, and will be further investigated.

Production of R-3-HB by B. cepacia using Acer saccharum WEH and Paulownia elongate WEH is significantly different (Figure 6). Almost no R-3-HB can be detected in the fermentation broth using Acer saccharum WEH during the whole process. On the other hand, production of $\mathrm{R}-3-\mathrm{HB}$ was detected in the medium using Paulownia elongate WEH during the third day of fermentation. The R-3-HB production started at day $3(50 \mathrm{~h})$, and then a significant increase to $14.2 \mathrm{~g} / \mathrm{L}$ was observed at day $4(76 \mathrm{~h})$. The highest HB centration reached was $16.8 \mathrm{~g} / \mathrm{L}$ at day $9(208 \mathrm{~h})$, which was then followed by a decreasing trend to $10.5 \mathrm{~g} / \mathrm{L}$ at day 12 (262 h).

\section{Investigation of R-3-HB production using simulated WEH}

Compared to Acer saccharum WEH, Paulownia elongate WEH has much more six carbon monosaccharides, mainly glucose and galactose, as well as a significant amount of oligosaccharides. Furthermore, the dilute acid hydrolysis process introduced extra chloride and nitrate ions into the Paulownia elongate WEH.

To investigate the metabolism that triggered the production of R-3-HB from Paulownia elongate WEH, we designed a series of batch fermentations. $50 \mathrm{~g} / \mathrm{L}$ glucose solution, $50 \mathrm{~g} / \mathrm{L}$ xylose solution, $50 \mathrm{~g} / \mathrm{L}$ xylose solution with extra nitrate ion, and $50 \mathrm{~g} / \mathrm{L}$ xylose solution with extra nitrate and chloride ions were all used to simulate the Paulownia elongate WEH as fermentation substrate. 


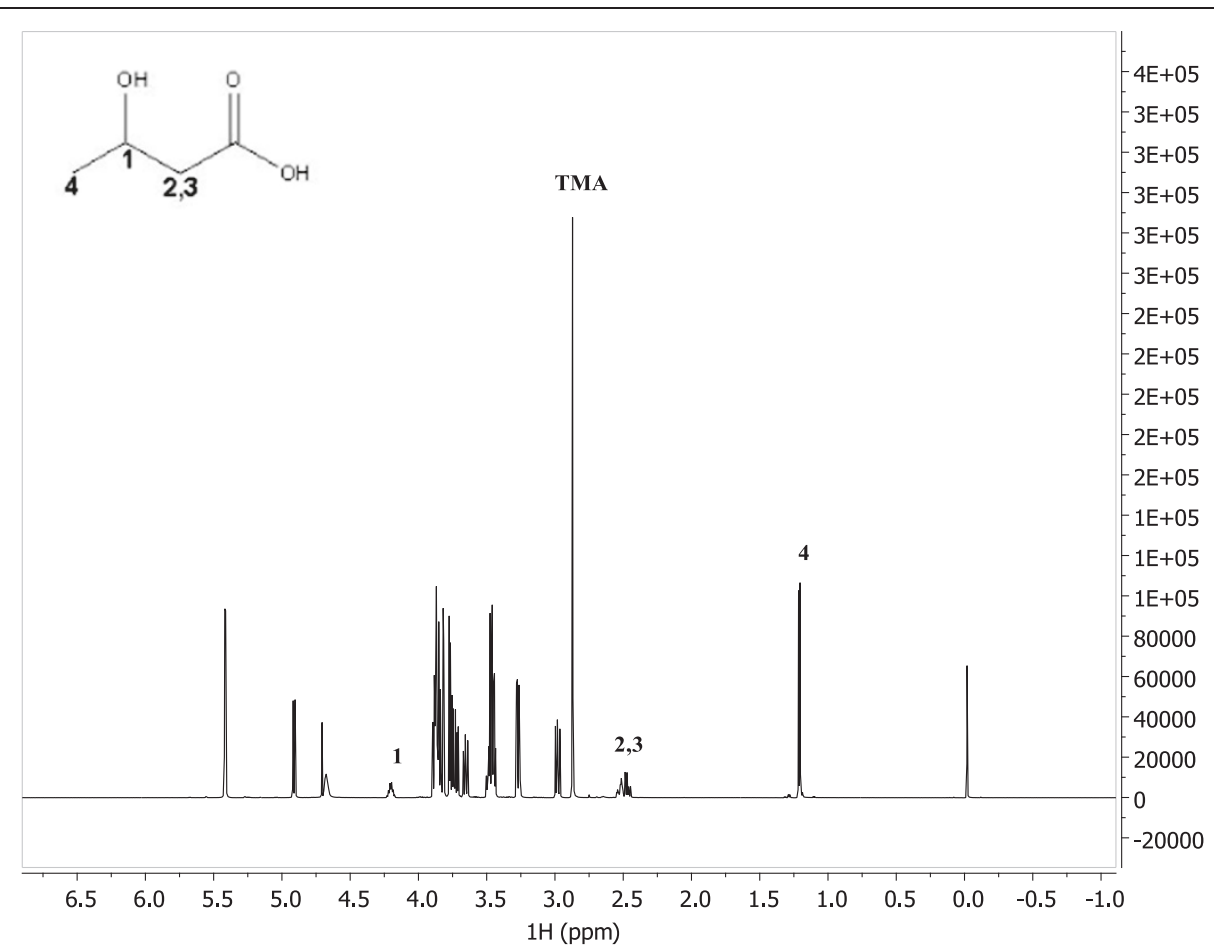

Figure 2 Proton NMR spectrum of reagent 3-HB.

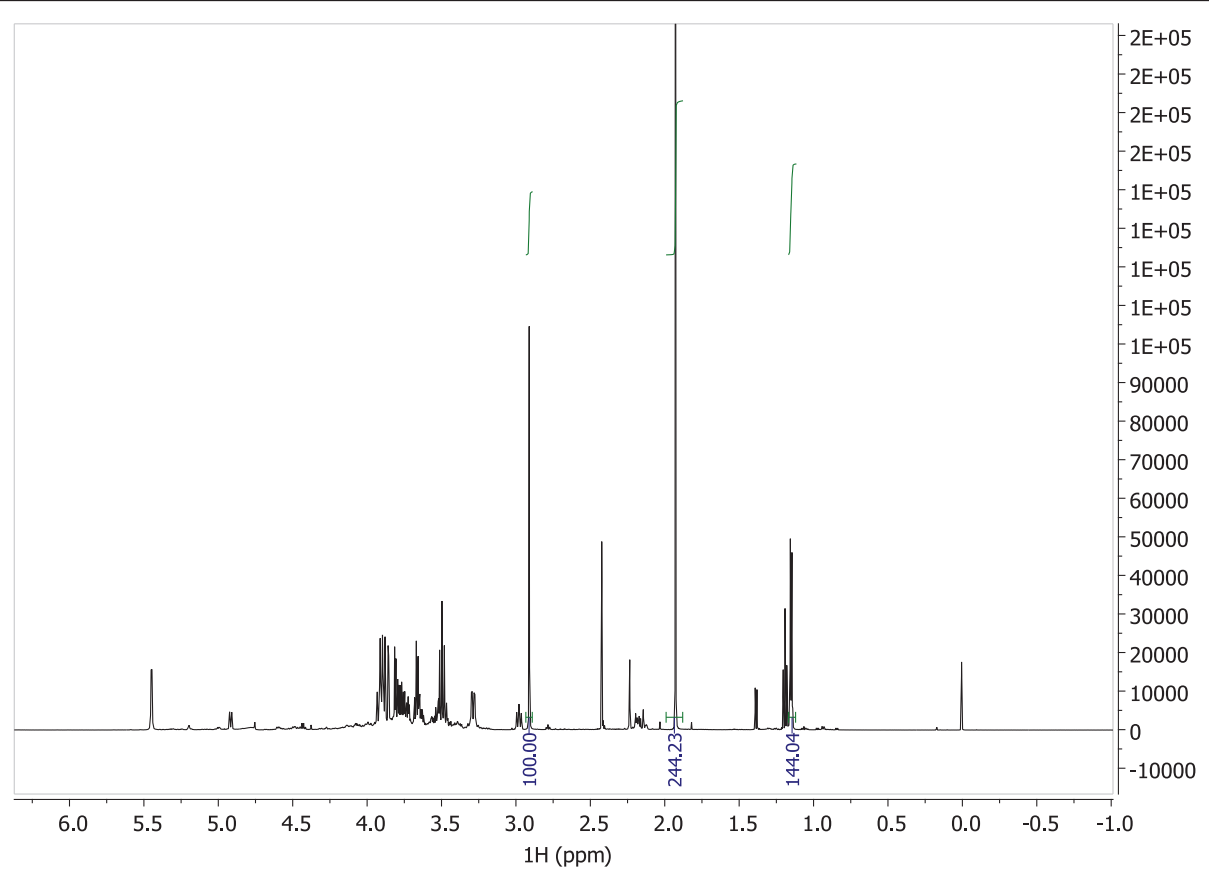

Figure 3 Proton spectrum of $B$. cepacia fermentation broth after 8 days of fermentation using Paulownia elongate WEH as substrate. 


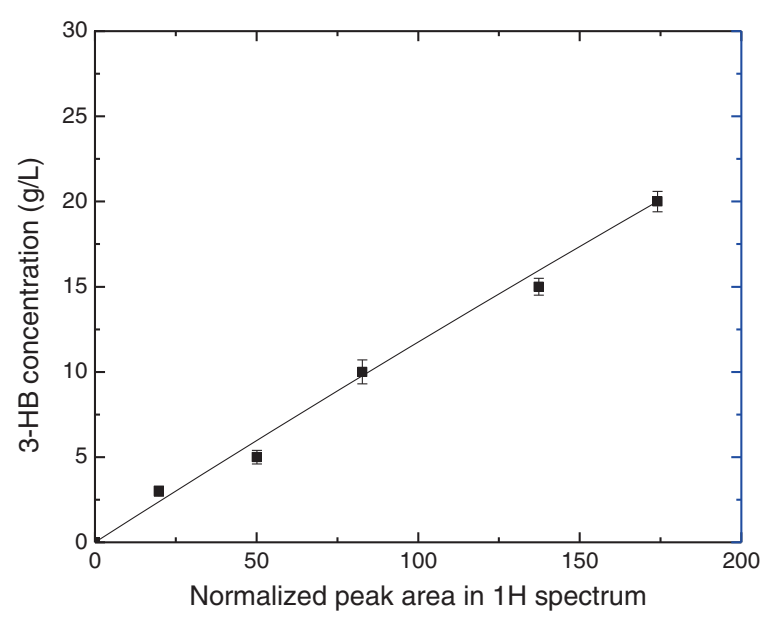

Figure 4 Standard curve of normalized peak area of 3-HB in proton spectrum corresponding to its concentration.

Results of fermentation using glucose solution and $x y-$ lose solution indicated that glucose as the fermentation substrate favors the production of $\mathrm{R}-3-\mathrm{HB}$ more than using xylose (Figure 7). Introduction of extra nitrate and chloride ions seems to have a positive influence on the extracellular accumulation of R-3-HB.

The maximum R-3-HB concentration achieved from simulated WEH was $5.9 \mathrm{~g} / \mathrm{L}$ from glucose. Using xylose as the fermentation substrate, highest $\mathrm{R}-3-\mathrm{HB}$ concentration achieved is $3.8 \mathrm{~g} / \mathrm{L}$ with extra nitrate and chloride ions. However, the extracellular concentration of R-3-HB reached $16.8 \mathrm{~g} / \mathrm{L}$ using Paulownia elongate WEH. Some other compounds in the Paulownia elongate WEH might also contribute to the high R-3-HB production.

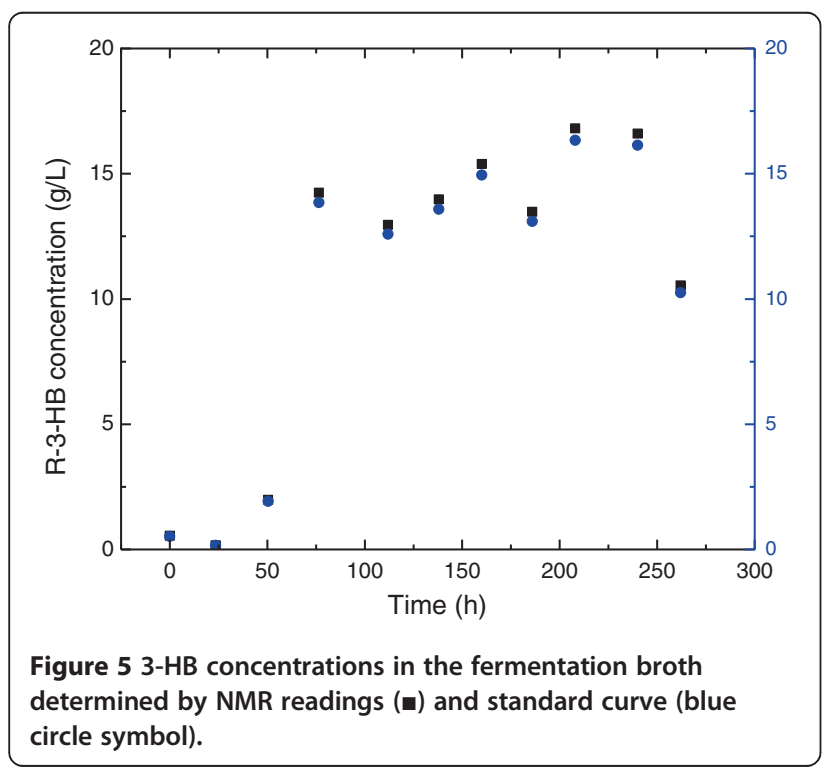

Biomass and PHB yield in B. cepacia fermentation

During the balanced growth phase, synthesis of PHA has been observed to occur simultaneously with cell growth and reproduction (Braunegg et al. 1998; Lee et al. 1999). However, the microbial PHA accumulation has been observed to increase significantly during imbalanced growth (Braunegg et al. 1998; Sudesh et al. 2000), such as nitrogen (Tappel et al. 2012), phosphorus (de Roo et al. 2002), oxygen (Lv et al. 2012), magnesium (Hu et al. 2011), or sulfate limited conditions with excess carbon (Braunegg et al. 1998; Riis and Mai 1988). PHA production has generally been understood as induced by lack of one or more key nutrient for growth.

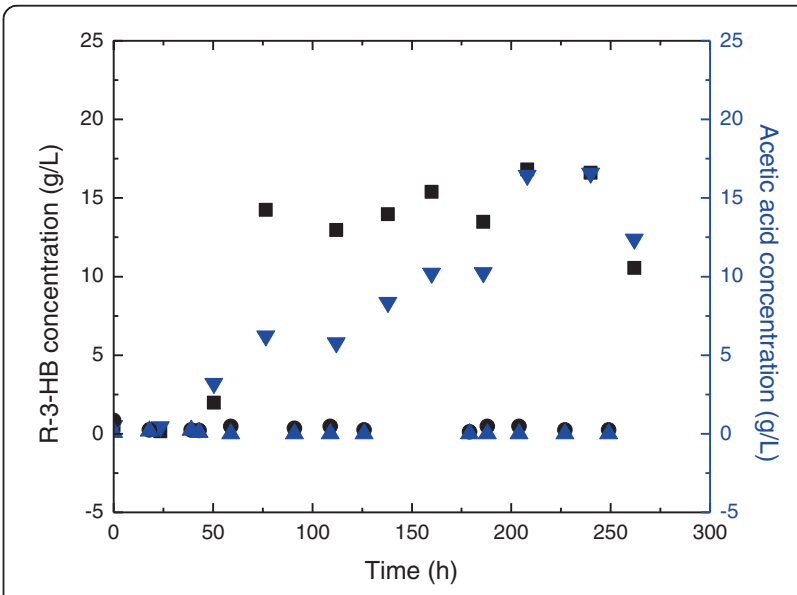

Figure 6 R-3-HB production during batch fermentation of B. cepacia using $50 \%$ Acer saccharum WEH or $50 \%$ Paulownia elongate WEH as substrate (R-3-HB in Acer saccharum WEH: •; R-3-HB in Paulownia elongate WEH: $\square$; Acetic acid in Acer saccharum WEH: blue up pointing triangle symbol; Acetic acid in Paulownia elongate WEH:blue down pointing triangle symbol). 


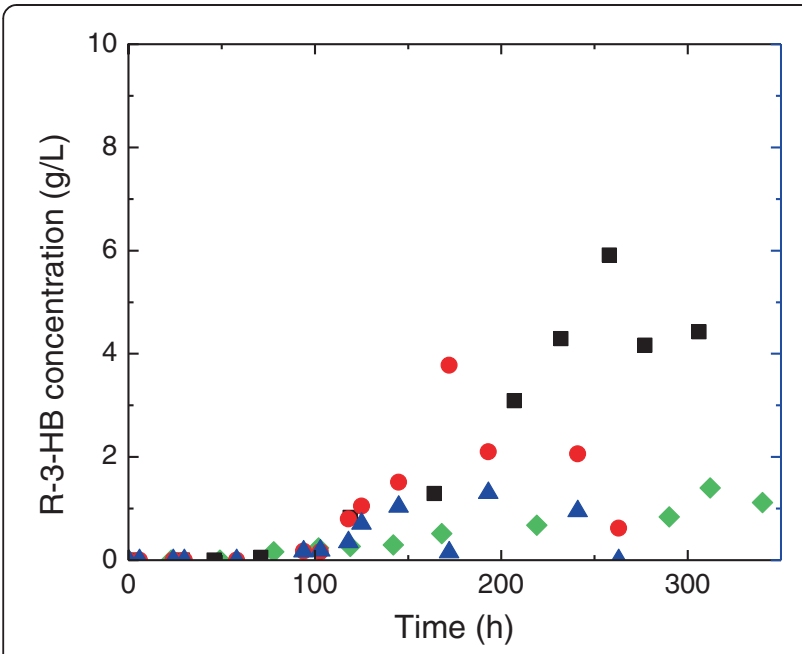

Figure 7 R-3-HB production during batch fermentation using $50 \mathrm{~g} / \mathrm{L}$ glucose or $50 \mathrm{~g} / \mathrm{L}$ xylose as substrate (xylose: green diamond symbol; glucose: $n$; xylose with extra nitrate and chloride ions red circle symbol; xylose with extra nitrate ion blue up pointing triangle symbol).

In this study, nitrogen limited culture condition was adapted in the batch fermentations. The biomass yield and PHB content in the harvested biomass from six batch fermentations using 50\% Acer saccharum WEH, $50 \%$ Paulownia elongate WEH, $50 \mathrm{~g} / \mathrm{L}$ glucose, $50 \mathrm{~g} / \mathrm{L}$ xylose, $50 \mathrm{~g} / \mathrm{L}$ xylose with extra nitrate ion, and $50 \mathrm{~g} / \mathrm{L}$ xylose with extra nitrate and chloride ions as substrate (Figures 8 and 9) respectively agreed with previous reported phenomenon that synthesis of PHA generally occur simultaneously with cell growth and reproduction (Braunegg et al. 1998; Lee et al. 1999). Another noteworthy phenomenon observed from these batch fermentations is that higher concentration of extracellular R-3-HB was detected from the fermentations with lower biomass and PHB yield, while less R-3-HB was produced from the fermentations with higher biomass and PHB yield, which confirms the mechanism proposed in Figure 10 that extracellular R-3-HB is generated by intracellular depolymerization of PHB followed by secretion. Using Acer saccharum WEH and xylose solution as substrate obtained the highest biomass and PHB yield, but produced negligible amount of R-3-HB monomers. On the other hand, high concentration of R-3-HB was produced from Paulownia elongate WEH with comparatively lower yield of biomass and polymers.

\section{Discussion}

NMR has been successfully used to identify and quantify HAs (Lee et al. 1999; Seebach et al. 2003). Furthermore, NMR can be used to identity and quantify HA along with other components in the fermentation broth, including monosaccharaides, oligomers, and other byproducts. NMR samples can be run in minutes, yielding both proton and 2D-HSQC spectrums from a single sample, while eliminating difficulty in solvent selection and standard solutions. It is a well acknowledged quantitative analysis method in chemistry (Shupe et al. 2012).

Currently, there are about 150 monomers that have been identified from the naturally produced PHAs (Steinbüchel and Valentin 1995). It has been well acknowledged that all of these monomers are in the (R)$(-)$-configuration if they have chiral center at the carbon connects to the hydroxyl group (Gangoiti et al. 2010; Lee 1996; Lee et al. 1999; Liu et al. 2007; Matsumoto et al. 2013; Park et al. 2004; Romano et al. 2005; Ruth et al. 2007; Steinbüchel and Valentin 1995).

It has been demonstrated that in many wild type bacteria, the in vivo biosynthesis and degradation of PHA simultaneously take place (Wang et al. 2013). A model of the cyclic metabolism of PHA is proposed and shown

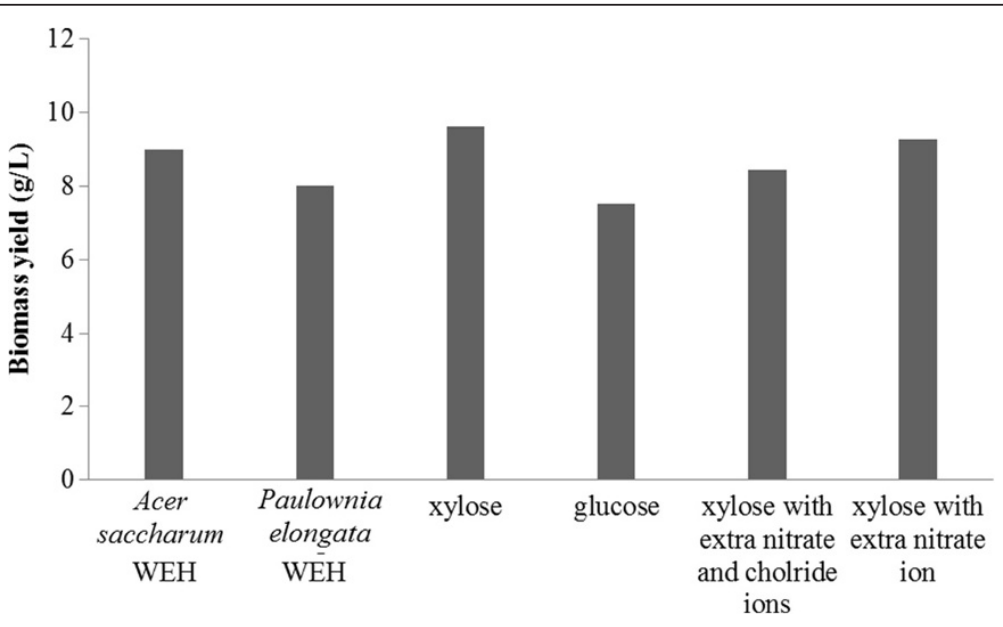

Figure 8 Biomass yield form batch fermentations. 


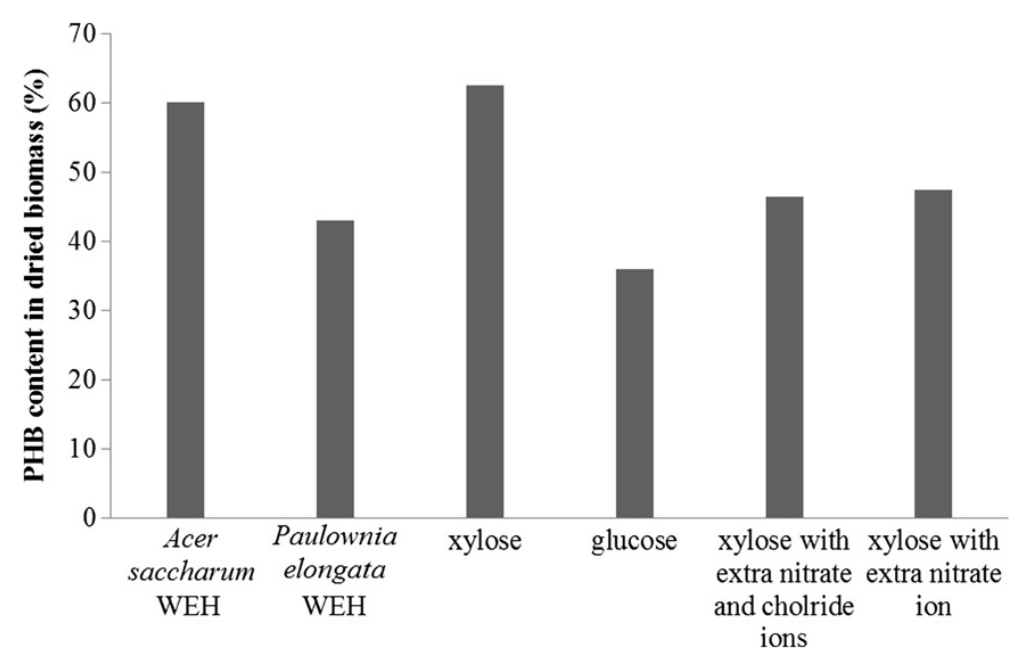

Figure 9 PHA content in dried biomass from batch fermentations.

in Figure 10 (Braunegg et al. 1998; Holmes 1985; Sudesh et al. 2000; Sudesh and Iwata 2008). From the metabolic pathways we can conclude that it is essential to increase or maintain a high activity of PHA depolymerase while lowering the activity of (R)-3-Hydroxylbutyrate dehydrogenase in order to increase the production of R-HAs (Shiraki et al. 2006; Tokiwa and Ugwu 2007; Yuan et al. 2008). There have been studies carried out on HA production through genetic engineering approach by overexpressing PHA depolymerase gene and/or repressing the (R)-3-Hydroxylbutyrate dehydrogenase gene (Holscher et al. 2010; Lee and Lee 2003; Shiraki et al. 2006; Ugwu et al. 2008).

Results of fermentations using six different medium showed that the concentration of R-3-HB in the fermentation broth increases at the expense of PHB yield, which confirms that R-3-HB is generated from the intracellular

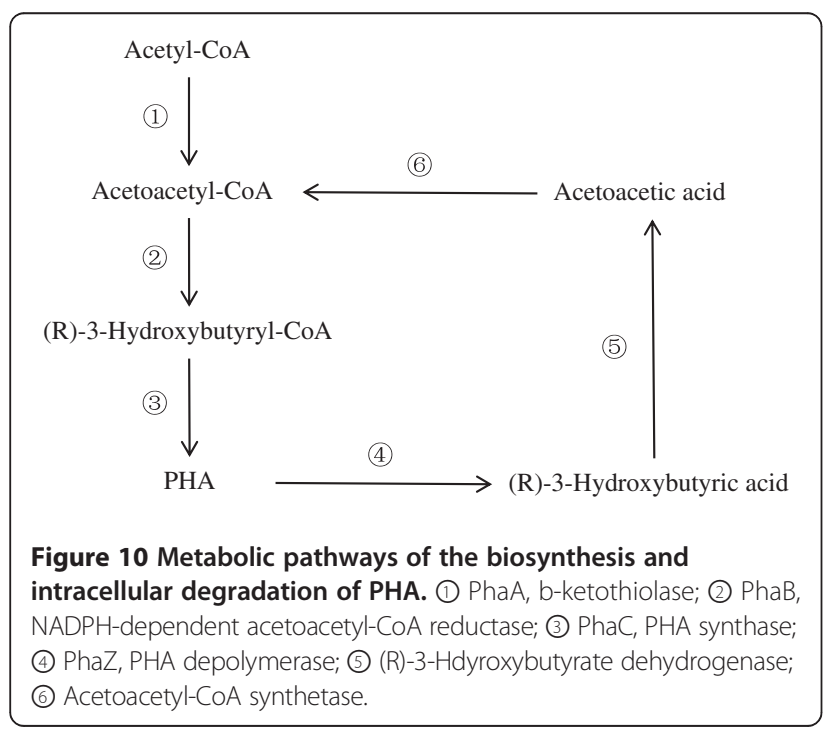

degradation of PHB. The mechanism of R-3-HB leakage into the fermentation broth would require further investigation. Biomass yield is higher in fermentation using $x y-$ lose as carbon substrate compared to glucose, which agrees with the phenomena of higher biomass yield in Acer saccharum WEH than in Paulownia elongate WEH because there is more xylose in Acer saccharum WEH while Paulownia elongate WEH has significant amount of glucose.

Previous reports have indicated that controlling $\mathrm{pH}$ of the fermentation medium can shift metabolic pathway to enhance in vivo depolymerization of PHAs and thus favor R-HAs production (Lee et al. 1999; Ren et al. 2005). Introducing successive aeration conditions has been reported to increase the intracellular degradation of PHB to (R)-3$\mathrm{HB}$, which was then secreted into the culture broth (Kawata et al. 2012). From this study, we could conclude that the composition of the fermentation substrate is also an important factor for R-HA production.

In good agreements with previous reports on HA production, it was found that the HA monomer secreted into the fermentation medium can be consumed by the cells again during fermentation (Lee et al. 1999). Therefore, it would be a more efficient strategy to produce HAs by removing the HA monomers continuously during the fermentation (Yuan et al. 2008).

In conclusion, production of $\mathrm{R}-\mathrm{HB}$ directly into the fermentation broth by suitable fermentation conditions enhances the prospect of biodegradable polymer PHA. High concentration $(16.8 \mathrm{~g} / \mathrm{L})$ of R-3-HB was produced by $B$. cepacia from Paulownia elongate WEH. The existence of oligosaccharides, six carbon monosaccharides such as glucose, as well as extra electrolytes such as nitrate and chloride ions in the fermentation medium has been observed to contribute to accumulation of R-3-HB 
in the fermentation supernatant. $\mathrm{R}-3-\mathrm{HB}$ is generated from the intracellular depolymerization of storage compound $\mathrm{PHB}$ followed by secretion into the fermentation broth. This study launched an innovative approach to efficiently and economically produce R-HA.

\section{Competing interests}

The authors declare that they have no competing interests.

\section{Acknowledgements}

The authors gratefully acknowledge the help by Mr. David Kiemle for assisting with the NMR analysis. Mr. C.D. Wood, Mr. Jipeng Yan and Mr. Yipeng Xie helped to generate the wood extract hydrolysates.

Received: 3 January 2014 Accepted: 6 January 2014

Published online: 18 March 2014

\section{References}

Bertrand JL, Ramsay BA, Ramsay JA, Chavarie C (1990) Biosynthesis of poly-betahydroxyalkanoates from pentoses by Pseudomonas pseudoflava. Appl Environ Microbiol 56(10):3133-3138

Braunegg G, Lefebvre G, Genser KF (1998) Polyhydroxyalkanoates, biopolyesters from renewable resources: Physiological and engineering aspects. $J$ Biotechnol 65(2-3):127-161

Buyondo JP, Liu SJ (2011) Lactic acid production by Lactobacillus pentosus from wood extract hydrolysates. J-for-J Sci Technol Forest Products Process 1 (3):38-47

Chen GQ (2009) A microbial polyhydroxyalkanoates (PHA) based bio- and materials industry. Chem Soc Rev 38(8):2434-2446

Chen GQ, Wu Q (2005) Microbial production and applications of chiral hydroxyalkanoates. Appl Microbiol Biotechnol 67(5):592-599

Choi J, Lee SY (1999) Factors affecting the economics of polyhydroxyalkanoate production by bacterial fermentation. Appl Microbiol Biotechnol 51(1):13-21

Chung A, Liu Q, Ouyang S-P, Wu Q, Chen G-Q (2009) Microbial production of 3-hydroxydodecanoic acid by pha operon and fadBA knockout mutant of Pseudomonas putida KT2442 harboring tesB gene. Appl Microbiol Biotechnol 83(3):513-519

de Roo G, Kellerhals MB, Ren Q, Witholt B, Kessler B (2002) Production of chiral R-3-hydroxyalkanoic acids and R-3-hydroxyalkanoic acid methylesters via hydrolytic degradation of polyhydroxyalkanoate synthesized by pseudomonads. Biotechnol Bioeng 77(6):717-722

Gangoiti J, Santos M, Llama MJ, Serra JL (2010) Production of chiral (R)-3hydroxyoctanoic acid monomers, Catalyzed by Pseudomonas fluorescens GK13 poly(3-hydroxyoctanoic acid) depolymerase. Appl Environ Microbiol 76(11):3554-3560

Holmes PA (1985) Applications of PHB - a microbially produced biodegradable thermoplastic. Phys Technol 16(1):32

Holscher T, Breuer U, Adrian L, Harms H, Maskow T (2010) Production of the chiral compound (R)-3-hydroxybutyrate by a genetically engineered methylotrophic bacterium. Appl Environ Microbiol 76(16):5585-5591

Hu D, Chung AL, Wu LP, Zhang X, Wu Q, Chen JC, Chen GQ (2011) Biosynthesis and characterization of polyhydroxyalkanoate block copolymer P3HB-b-P4HB. Biomacromolecules 12(9):3166-3173

Kawata Y, Kawasaki K, Shigeri Y (2012) Efficient secreted production of (R)-3hydroxybutyric acid from living Halomonas sp KM-1 under successive aerobic and microaerobic conditions. Appl Microbiol Biotechnol 96(4):913-920

Keenan TM, Tanenbaum SW, Stipanovic AJ, Nakas JP (2004) Production and characterization of poly- $\beta$-hydroxyalkanoate copolymers from Burkholderia cepacia utilizing xylose and levulinic acid. Biotechnol Prog 20(6):1697-1704

KI M, Okei T, Honma I, Ooi T, Aoki H, Taguchi S (2013) Efficient (R)-3hydroxybutyrate production using acetyl CoA-regenerating pathway catalyzed by coenzyme A transferase. Appl Microbiol Biotechnol 97(1):205-210

Lee SY (1996) Bacterial polyhydroxyalkanoates. Biotechnol Bioeng 49(1):1-14

Lee SY, Lee Y (2003) Metabolic engineering of Escherichia coli for production of enantiomerically pure (R)-(-)-hydroxycarboxylic acids. Appl Environ Microbiol 69(6):3421-3426

Lee SH, Park OJ (2009) Uses and production of chiral 3-hydroxy-gamma-butyrolactones and structurally related chemicals. Appl Microbiol Biotechnol 84(5):817-828
Lee SY, Lee Y, Wang F (1999) Chiral compounds from bacterial polyesters: Sugars to plastics to fine chemicals. Biotechnol Bioeng 65(3):363-368

Liu S (2012) Woody biomass conversion: sustainability and water-based processes. J Bioproc Eng Bioref 1(1):6-32

Liu Q, Ouyang SP, Chung A, Wu Q, Chen GQ (2007) Microbial production of R-3hydroxybutyric acid by recombinant $E$. coli harboring genes of $p h b A$, phbB, and tesB. Appl Microbiol Biotechnol 76(4):811-818

Lv SH, Gong R, Ma YF (2012) Structure and properties of the graft copolymer of starch and p-hydroxybenzoic acid using horseradish peroxidase. Polym Adv Technol 23(10):1343-1349

Park SJ, Lee SY, Lee Y (2004) Biosynthesis of (R)-3-hydroxyalkanoic acids by metabolically engineered Escherichia coli. Appl Biochem Biotechnol 113:373-379

Ren Q, Grubelnik A, Hoerler M, Ruth K, Hartmann R, Felber H, Zinn M (2005) Bacterial poly(hydroxyalkanoates) as a source of chiral hydroxyalkanoic acids. Biomacromolecules 6(4):2290-2298

Ren Q, Ruth K, Thoeny-Meyer L, Zinn M (2010) Enatiomerically pure hydroxycarboxylic acids: current approaches and future perspectives. Appl Microbiol Biotechnol 87(1):41-52

Riis V, Mai W (1988) Gas chromatographic determination of poly- $\beta$ hydroxybutyric acid in microbial biomass after hydrochloric acid propanolysis. J Chromatogr A 445(C):285-289

Romano A, van der Plas LHW, Witholt B, Eggink G, Mooibroek H (2005) Expression of poly-3-(R)-hydroxyalkanoate (PHA) polymerase and acyl-CoAtransacylase in plastids of transgenic potato leads to the synthesis of a hydrophobic polymer, presumably medium-chain-length PHAs. Planta 220(3):455-464

Ruth K, Grubelnik A, Hartmann R, Egli T, Zinn M, Ren Q (2007) Efficient production of (R)-3-hydroxycarboxylic acids by biotechnological conversion of polyhydroxyalkanoates and their purification. Biomacromolecules 8(1):279-286

Sandoval A, Arias-Barrau E, Bermejo F, Canedo L, Naharro G, Olivera E, Luengo J (2005) Production of 3-hydroxy-n-phenylalkanoic acids by a genetically engineered strain of Pseudomonas putida. Appl Microbiol Biotechnol 67(1):97-105

Seebach D, Beck AK, Breitschuh R, Job K (1992) Direct Degradation of the Biopolymer Poly[(R)-3-Hydroxybutyric Acid] to (R)-3-Hydroxybutanoic Acid and its Methyl Ester. Org Synth 71:39-47

Shiraki M, Endo T, Saito T (2006) Fermentative production of (R)-(-)-3hydroxybutyrate using 3-hydroxybutyrate dehydrogenase null mutant of Ralstonia eutropha and recombinant Escherichia coli. J Biosci Bioeng 102(6):529-534

Shupe AM, Liu SJ (2012a) Effect of agitation rate on ethanol production from sugar maple hemicellulosic hydrolysate by Pichia stipitis. Appl Biochem Biotechnol 168(1):29-36

Shupe AM, Liu SJ (2012b) Ethanol fermentation from hydrolysed hot-water wood extracts by pentose fermenting yeasts. Biomass Bioenergy 39:31-38

Shupe AM, Kiemle DJ, Liu S (2012) Quantitative 2D HSQC NMR analysis of mixed wood sugars in hemicellulosic hydrolysate fermentation broth. J Bioproc Eng Bioref 1(1):93-100

Steinbüchel A, Lütke-Eversloh T (2003) Metabolic engineering and pathway construction for biotechnological production of relevant polyhydroxyalkanoates in microorganisms. Biopolymers 16(2):81-96

Steinbüchel A, Valentin HE (1995) Diversity of bacterial polyhydroxyalkanoic acids. FEMS Microbiol Lett 128(3):219-228

Sudesh K, Iwata T (2008) Sustainability of biobased and biodegradable plastics. Clean-Soil Air Water 36(5-6):433-442

Sudesh K, Abe H, Doi Y (2000) Synthesis, structure and properties of polyhydroxyalkanoates: biological polyesters. Prog Polym Sci 25(10):1503-1555

Sun ZJ, Liu SJ (2012) Production of n-butanol from concentrated sugar maple hemicellulosic hydrolysate by Clostridia acetobutylicum ATCC824. Biomass Bioenergy 39:39-47

Tappel RC, Kucharski JM, Mastroianni JM, Stipanovic AJ, Nomura CT (2012) Biosynthesis of poly (R)-3-hydroxyalkanoate copolymers with controlled repeating unit compositions and physical properties. Biomacromolecules 13(9):2964-2972

Tokiwa Y, Ugwu CU (2007) Biotechnological production of (R)-3-hydroxybutyric acid monomer. J Biotechnol 132(3):264-272

Ugwu CU, Tokiwa Y, Aoyagi H, Uchiyama H, Tanaka H (2008) UV mutagenesis of Cupriavidus necator for extracellular production of (R)-3-hydroxybutyric acid. J Appl Microbiol 105(1):236-242

Wang Y, Liu S (2013) Polyhydroxyalkanoates (PHAs): biosynthesis, production and recovery. J Bioproc Eng Bioref 2(1):61-72 
Wang Y, Sun Z, Shupe A, Liu S (2012) Influence of oxygen mass transfer on the fermentation behavior of Burkholderia cepacia for polyhydroxyalkanoates (PHAs) production utilizing wood extract hydrolysate (WEH). J Bioproc Eng Bioref 1(2):169-175

Wang BQ, Sharma-Shivappa RR, Olson JW, Khan SA (2013) Production of polyhydroxybutyrate (PHB) by Alcaligenes latus using sugarbeet juice. Ind Crop Prod 43:802-811

Yan J, Joshee N, Liu S (2013) Kinetics of the hot water extraction of Paulownia Elongata woodchips. J Bioproc Eng Bioref 2(1):1-10

Yuan M-Q, Shi Z-Y, Wei X-X, Wu Q, Chen S-F, Chen G-Q (2008) Microbial production of medium-chain-length 3-hydroxyalkanoic acids by recombinant Pseudomonas putida KT2442 harboring genes fadL, fadD and phaZ. FEMS Microbiol Lett 283(2):167-175

doi:10.1186/s13568-014-0028-9

Cite this article as: Wang and Liu: Production of (R)-3-hydroxybutyric acid by Burkholderia cepacia from wood extract hydrolysates. AMB Express 2014 4:28.

\section{Submit your manuscript to a SpringerOpen ${ }^{\circ}$ journal and benefit from:}

- Convenient online submission

- Rigorous peer review

- Immediate publication on acceptance

- Open access: articles freely available online

- High visibility within the field

- Retaining the copyright to your article

Submit your next manuscript at $\gg$ springeropen.com 